\title{
Engagement in Nonbiomedical Practices for Fertility Enhancement: A Scoping Review
}

\author{
Carie Muntifering Cox, PhD, MPH and Courtney L. Johnson, MPH, MFA
}

\begin{abstract}
Objectives: This scoping review aims to (1) describe the scope and coverage of the body of literature on nonbiomedical practices used for enhancing fertility, (2) summarize and map the existing evidence on the extent and types of nonbiomedical practices used, and (3) examine how the research on this topic has been conducted with particular attention paid to how participants are asked about their use of nonbiomedical practices for fertility enhancement.

Design: We conducted a scoping review by which four databases were searched (PubMed, Psychinfo, Socindex, and CINHAL). Articles were screened for inclusion by two researchers through a title and abstract screening followed by a full-text screening. Data were extracted from included articles and results summarized and compared across studies and regions.

Results: A total of 32 studies were identified from 16 countries in five regions of the world. The majority of studies were clinic-based, primarily recruiting participants from fertility clinics in urban and suburban areas. More than half of the studies included only women, a few studies included only men, and the remainder of studies included both men and women. Most quantitative studies reported the prevalence of nonbiomedical practice use, which ranged from $8 \%$ to $83 \%$; however, there was variation across studies regarding the time frame for which these percentages were derived. Few studies reported on other measures of the extent of use such as duration or frequency. A variety of nonbiomedical practices were identified with biologically based treatments, particularly herbal medicine use, being the most common followed by religious and spiritual interventions. Regional differences were identified. Variation in the wording and format of the question(s) used in survey instruments asking participants about their use of nonbiomedical practices exists and may impact participants' reporting of use.

Conclusions: Infertility affects millions of individuals worldwide often with severe social, emotional, and financial consequences. To enhance fertility and, in some cases, overcome infertility, many individuals and couples are engaging in a variety of nonbiomedical practices. This scoping review describes the scope and nature of the existing literature on the use of nonbiomedical practices for fertility enhancement and highlights important gaps and limitations in the conduct of this research. A more comprehensive and inclusive investigation of nonbiomedical practices for enhancing fertility is needed to improve our understanding of how individuals and couples are managing infertility, identify educational and counseling needs, and to improve research related to effectiveness and safety of nonbiomedical practices.
\end{abstract}

Keywords: fertility, subfertility, fertility enhancement, complementary and alternative medicine, nonbiomedical practices, lifestyle modification

Department of Public Health, St. Catherine University, St. Paul, MN, USA. 


\section{Introduction}

G LOBALLY, AN ESTIMATED $15 \%$ of couples are infertile, ${ }^{1}$ with considerable variation within and between countries. $^{2,3}$ The social, psychological, and economic consequences of infertility can be devastating and often include social isolation, stigma, feelings of guilt and shame, depression, financial hardship due to treatment costs and, in some settings, loss of inheritance, ${ }^{4}$ and a decrease in overall quality of life and well-being. ${ }^{5}$ These consequences, often coupled with an intense desire to have a/another child, can make couples with infertility desperate for treatment. This point is further supported by multiple studies noting that individuals experiencing infertility report a willingness to "try anything', to conceive. ${ }^{6-8}$

In pursuit of conception, many individuals turn to nonbiomedical practices, which, for the purposes of this scoping review, we define as any practice or behavior that falls outside of conventional biomedicine and is used to enhance fertility. Some choose to use nonbiomedical practices in combination with conventional biomedicine, including assisted reproductive technologies (ART), ${ }^{9}$ whereas others choose to use it as an alternative to ART, often due to a lack of access, exorbitant costs, and/or a lack of success with previous ART cycles. ${ }^{7,9-12}$ Perceived beliefs about the causes of infertility $^{7,13}$ and/or a positive and trusting attitude toward nonbiomedical practices and practitioners also influence one's desire to engage in these practices. ${ }^{9,13,14}$ Additionally, individuals often receive encouragement and/or pressure from family and friends to seek out nonbiomedical practices when unable to conceive. ${ }^{11,15-17}$

Engagement in nonbiomedical practices is often done in the absence of scientific evidence on the effectiveness or safety of these practices due to a lack of research. ${ }^{18,19} \mathrm{~A}$ recent scoping review conducted by Miner et al. ${ }^{19}$ on the effectiveness of complementary and alternative medicine (CAM) on fertility found that acupuncture has been the most rigorously studied practice but the evidence is inconclusive as was the case for Ayurveda and Chinese Herbal Medicine. For the other seven practices examined in the review, there was a lack of quality scientific evidence that these practices improved fertility. ${ }^{19}$ Despite an overall lack of evidence on the effectiveness of nonbiomedical practices for enhancing fertility, a 2011 literature review by Rayner et al. ${ }^{9}$ found prevalence rates of use among women ranging from $29 \%$ to $91 \%$, This large range in prevalence demonstrates widespread use but also highlights significant variation across studies, which may be a reflection of true differences in prevalence or of differences in how the research was conducted. Understanding these differences is important for establishing the level of comparability across studies and for accurately interpreting findings.

According to the 2011 review, women are engaging in a wide variety of nonbiomedical practices to enhance their fertility. These practices range from minor behavior modifications such as changes in attire to entire medical systems such as Traditional Chinese Medicine, with the most common practices being herbal medicines, acupuncture, and nutritional advice and supplementation. ${ }^{9}$ The range of nonbiomedical practices identified in the review demonstrates the heterogeneous and expansive nature of these practices and raises questions about if and how researchers are accurately and comprehensively capturing participants' use of these practices in studies that seek to identify the extent and type of nonbiomedical practices employed for fertility enhancement.

Given the high burden of infertility and the widespread use of nonbiomedical practices for managing this condition, it is essential that we curate a better understanding of the extent, nature, and conduct of research on this topic. The 2011 literature review by Rayner et al. ${ }^{9}$ provides an important foundation for this work; however, there is a need to update these findings with more recent literature, expand the review to include men and women, and examine the evidence and conduct of research in a more in-depth and systematic manner. As such, we conducted a systematic scoping review to examine the breadth and depth of research on the practices people employ outside of conventional medicine to enhance their fertility. Specifically, we aimed to (1) describe the scope and coverage of the body of literature on nonbiomedical practices used for enhancing fertility, (2) summarize and map the existing evidence on the extent and types of nonbiomedical practices used, and (3) examine how the research on this topic has been conducted with particular attention paid to how participants are asked about their use of nonbiomedical practices for fertility enhancement. Focusing on both what and how information is being collected and reported allowed us to describe the current state of knowledge, assess comparability of findings across studies, and identify existing gaps. Improving the research on nonbiomedical practice use for fertility enhancement can lead to a more holistic understanding of how individuals and couples are managing infertility, improved assessment of associations between nonbiomedical practice use and predictor and outcome variables, identification of educational and counseling needs, and prioritization of nonbiomedical practices for effectiveness trials.

\section{Materials and Methods}

We conducted a scoping review of peer-reviewed publications on nonbiomedical practices used to enhance fertility. Scoping reviews are a newer approach to evidence synthesis with its key framework developed in 2005 by Arksey and O'Malley, ${ }^{20}$ with further guidance provided by Levac et al. in $2010,{ }^{21}$ Peters et al. in $2015,{ }^{22}$ and Munn et al. in 2018. ${ }^{23}$ As described by Munn et al., ${ }^{23}$ "scoping reviews are an ideal tool to determine the scope or coverage of a body of literature on a given topic and give clear indication of the volume of literature and studies available as well as an overview (broad or detailed) of its focus...They can report on the types of evidence that address and inform practice in the field and the way the research has been conducted." Based on this description and its alignment with our research aims, we deemed the scoping review methodology the most appropriate approach for our review.

\section{Identifying relevant studies}

We conducted searches in PubMed, Psychinfo, Socindex, and CINAHL using a broad set of search terms (Table 1). Medical subject headings (MeSH) terms were used when searching PubMed and, when available, equivalent subject headings were used when searching CINAHL. The combination of our search terms captured studies on both 
Table 1. Categorical Organization of Search Terms

Category Search terms combined with AND

\begin{tabular}{|c|c|}
\hline $\begin{array}{l}\text { Nonbiomedical } \\
\text { practices }\end{array}$ & $\begin{array}{l}\text { acupuncture OR alternative medicine OR alternative therapies OR alternative therapy OR ayurvedic } \\
\text { OR complementary medicine OR complementary therapies* OR exercise OR diet OR folklore OR } \\
\text { healthy lifestyle* OR herbal medicine OR herbs OR hypnosis OR massage OR medicinal (plants)* } \\
\text { OR medicinal (teas) OR meditation OR misconceptions OR myths OR naturopathy OR prayer OR } \\
\text { religion OR reproductive behavior OR sexual behavior OR sexual position OR shamanism OR } \\
\text { spiritual therapies* OR traditional medicine OR witchcraft OR yoga }\end{array}$ \\
\hline Reproduction & fertility OR infertility OR subfertility OR sterility \\
\hline
\end{tabular}

*MeSH used in addition to key words.

$\mathrm{MeSH}$, medical subject headings.

enhancing fertility and preventing pregnancy and, as a result, our search generated a large number of irrelevant studies on contraception that were eliminated during the title and abstract screening phase as reflected in the flowchart in Figure 1. Searches were limited to peer-reviewed articles written in English between January 2000 and November 2018. The latter restriction ensured that the results of the review and subsequent recommendations reflected current practices and trends. Search results were uploaded to RefWorks and duplicates removed. We also hand searched the reference list of two literature reviews for additional studies.

\section{Selecting studies}

Both members of the research team independently screened articles for inclusion in a title and abstract screening phase followed by a full-text screening phase. Original quantitative and qualitative research articles were considered. Randomized controlled trials were excluded given that participants would be randomized to certain practices and thus not a valid indication of their organic behavior. For a study to be included, participants had to have been explicitly asked about their personal use of nonbiomedical practices for fertility enhancement. We excluded articles that focused on a single practice.

\section{Charting the data}

We developed a data extraction tool to gather general information about the article, methods used, and sample characteristics as well as information specific to our research aims, such as the different types of nonbiomedical practices used and the extent of use. We also extracted phrasing and response options of relevant questions in survey instruments of quantitative studies. We obtained the exact wording of the relevant questions for eight studies either directly from
FIG. 1. Flow chart of search strategy and selection of articles.

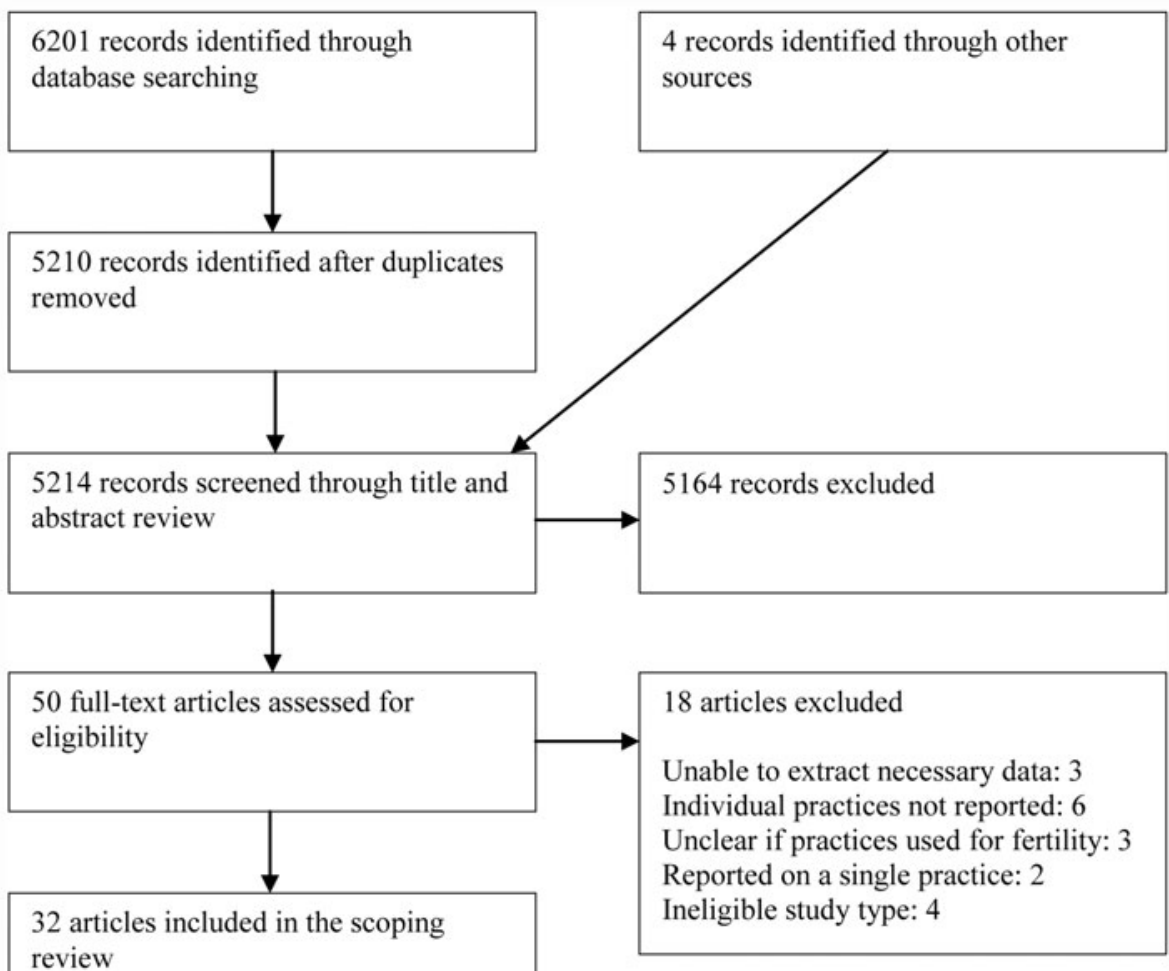

review 
the article or through email correspondence with the author. ${ }^{8,14,17,24-28}$ Ten additional studies provided a level of detail about their questions that allowed us to draw conclusions for certain aspects of this subanalysis. ${ }^{15,16,29-36} \mathrm{We}$ did not conduct a formal assessment of the quality of included studies given that the intent of a scoping review is to provide an overview of all existing literature regardless of quality and, as such, this type of assessment is generally not conducted as part of a scoping review. ${ }^{22}$

\section{Collating, summarizing, and reporting results}

We report key study characteristics to describe the scope and coverage of the literature reviewed. We then identified the different measures used to report the extent of use of nonbiomedical practices and summarized these findings. Finally, we mapped the use of specific nonbiomedical practices and how participants were asked about their use of these practices to a classification structure and report notable differences by region. We used the five category classification structure for CAM developed by the National Center for Complementary and Alternative Medicine (NCCAM), ${ }^{37}$ now known as the National Center for Complementary and Integrative Health (NCCIH), plus two additional categories that were reflected in our data. Altogether, the seven categories include: (1) Alternative medical systems, (2) Mind/ body interventions, (3) Biologically based treatments, (4) Manipulative and body-based methods, (5) Energy therapies, (6) Religious and spiritual interventions, and (7) Lifestyle modification. The NCCAM classification structure is widely used, ${ }^{37,38}$ including in a recent scoping review on the effectiveness of CAM during fertility treatments, ${ }^{19}$ and thus will be familiar to most researchers in this field. Furthermore, many studies in our review provided limited details when reporting on specific practices, making the NCCAM classification structure, which is based on types of therapies, amenable to our data, since most practices can be categorized within this structure with limited information. Although there is overlap between categories, we assigned each practice to a single category guided by the Cochrane Collaboration $^{38}$ and the MSD Manual on CAM. ${ }^{39}$ We followed the PRISMA-ScR checklist when developing this article. $^{40}$

\section{Results}

As shown in Figure 1, a total of 6201 records were identified by searching four databases, which, after removing duplicates, resulted in 5210 unique records. An additional four unique records were identified when hand searching reference lists of related literature reviews and when preparing the background section. Following the title and abstract screening, 50 articles were screened in full, of which 18 articles were excluded due to not meeting at least one inclusion criteria. In total, 32 articles were included in our scoping review.

\section{Characteristics of included studies}

The majority of studies in our review were cross-sectional $(n=19)$, eight were qualitative, and five were prospective cohort (Table 2). All articles were published between 2000 and 2018 with the majority published in or after 2010 $(n=21)$. Using World Bank country classifications ${ }^{41}$ five of the seven world regions were represented in our review, including North America $(n=7)$, Europe and Central Asia $(n=9)$, Middle East and North Africa $(n=6)$, East Asia and Pacific $(n=7)$, and South Asia $(n=3)$. Sixteen unique countries were represented with the most studies originating from Turkey $(n=6)$, the United States $(n=5)$, and Australia $(n=4)$ (Fig. 2). The vast majority of studies were clinic based, primarily recruiting subfertile participants from urban and suburban fertility clinics.

Over half of the studies included only women, 3 studies included only men, and 11 studies included both men and women either independently and/or as couples (Table 2). Most studies did not indicate sexual orientation of participants, although some did restrict their sample to heterosexual and/or married participants in countries that do not recognize same-sex marriage. Only one study mentioned inclusion of a participant with a same-sex partner. ${ }^{6}$

\section{Extent of use of nonbiomedical practices}

Twenty quantitative studies provided overall prevalence of the use of nonbiomedical practices for enhancing fertility. Prevalence ranged from $8 \%^{24}$ to $83 \%^{35}$ with most falling within $30 \%$ and $70 \%$. The time frame under investigation varied by study with some reporting on ever-use while others reporting on use during a specific period or exact point in time (Table 2); however, no clear patterns of how this impacted prevalence emerged. In the four studies reporting prevalence for both men and women, ${ }^{17,25,33,42}$ prevalence was lower among men in all but one study. ${ }^{17}$

Only four studies reported on frequency of use, with varying degree of specificity. ${ }^{28,29,32,43}$ For example, Ozkan et al. ${ }^{43}$ reported the percent that "never used," "used for awhile," and "regularly used" a given practice, whereas Dehghan et al. ${ }^{32}$ reported the range, mean, and standard deviation for the number of times a practice was used in the past year. Four studies reported on changes in the use of nonbiomedical practices. ${ }^{16,24,28,29}$ For example, Domar et al. ${ }^{28}$ compared use of various practices during an in vitro fertilization (IVF) cycle to use during the past month and past 5 years. No studies reported on duration of use.

It was evident from the data presented in most studies that some participants were engaging in multiple nonbiomedical practices to enhance fertility; however, only three studies presented data on the number of practices per participant. ${ }^{31-33}$ Among participants who reported using nonbiomedical practices, an average of 1.47 practices were reported in Denmark ${ }^{31}$ and 1.78 in Israel. ${ }^{33}$ Seven percent and $35 \%$ of participants reported using three of more practices in Denmark ${ }^{31}$ and Iran, ${ }^{32}$ respectively. Additionally, a qualitative study conducted in Australia reported that most women in the study, all of whom were CAM users, were consulting a variety of CAM practitioners. ${ }^{6}$

\section{Types of nonbiomedical practices}

As described in the methods section, we mapped the types of nonbiomedical practices reported to a seven-category classification structure, which we present below by summarizing the most common practices reported within and across studies for each category. We use the same classification structure in Table 3, which provides a more 


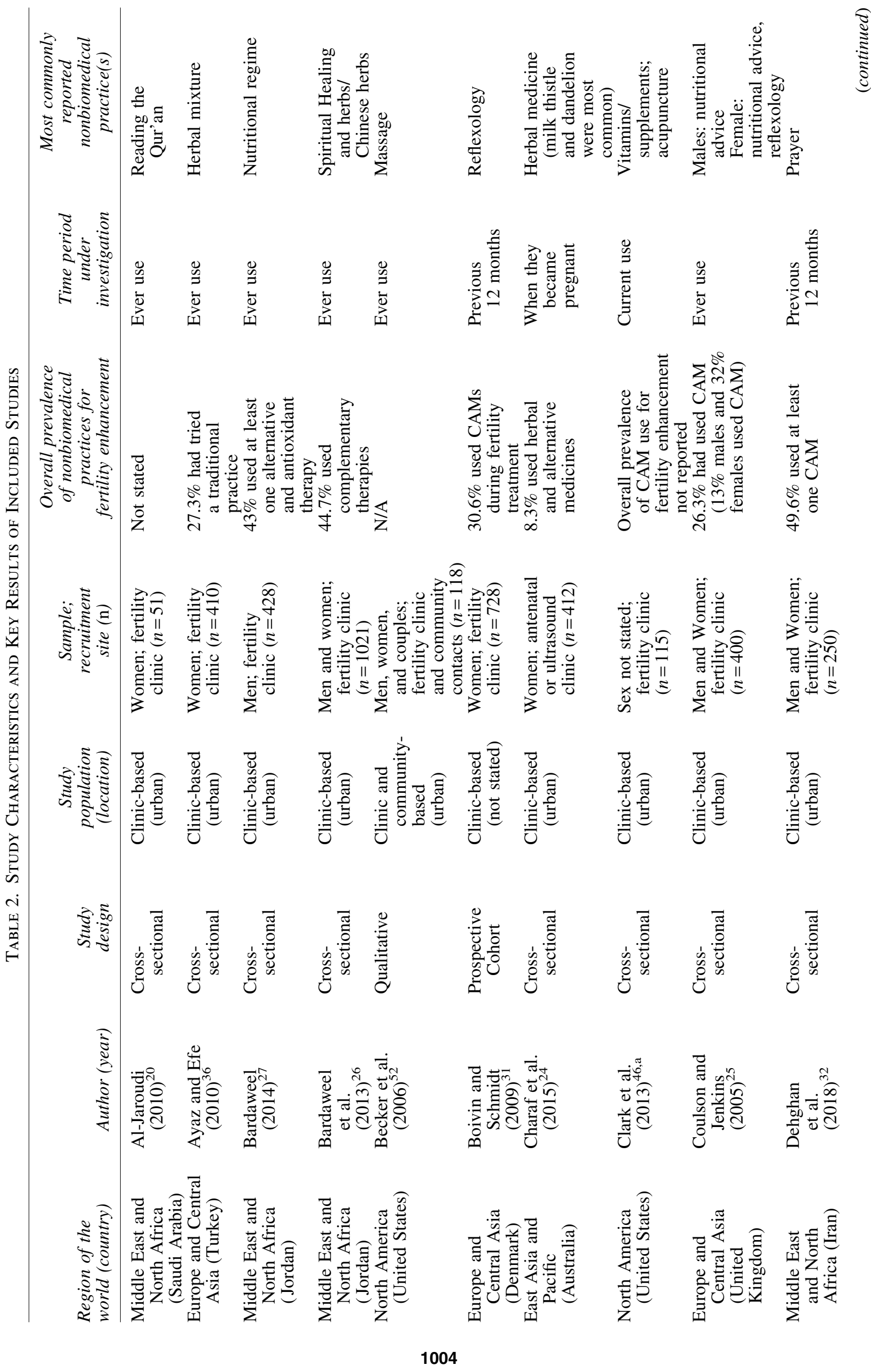




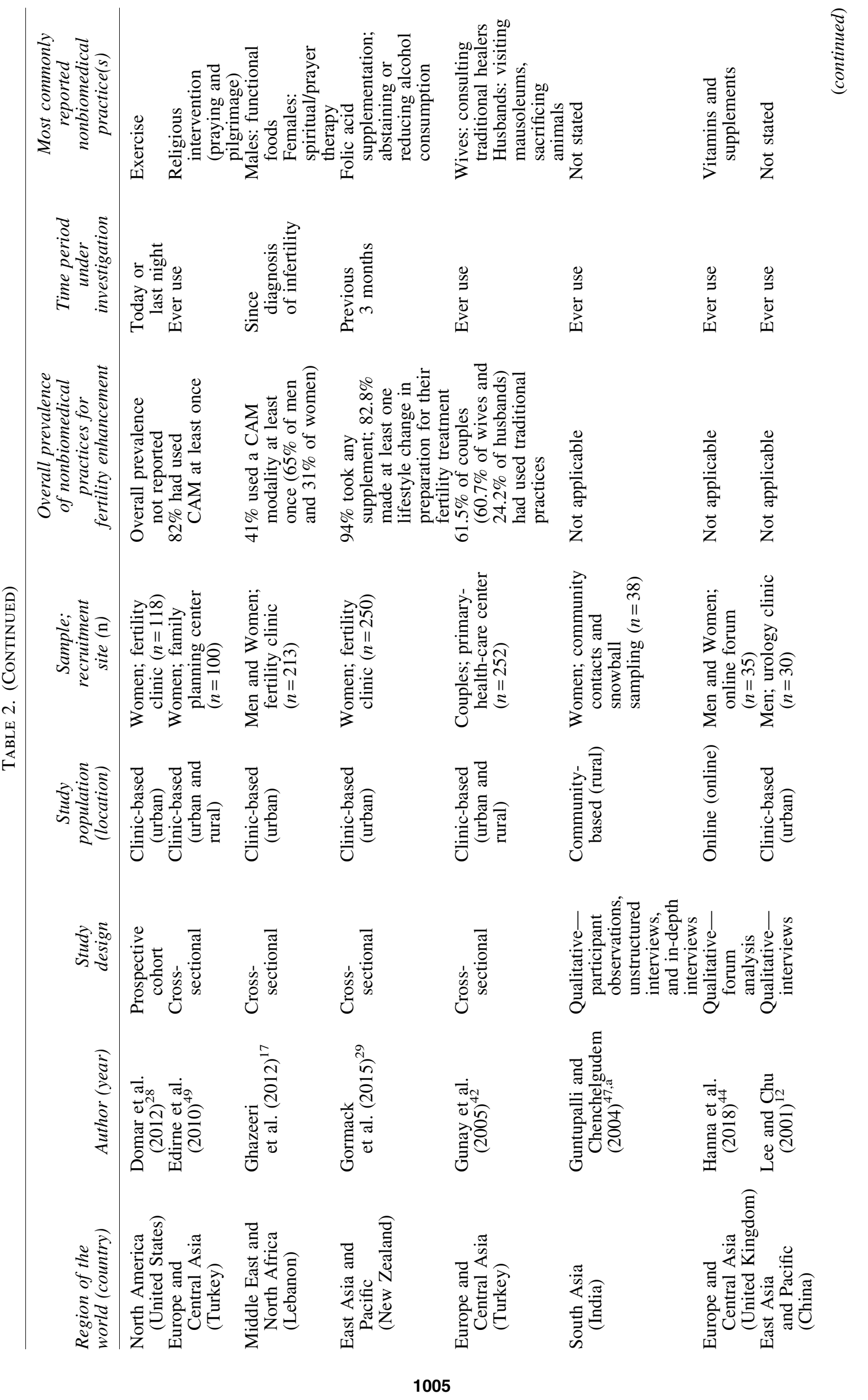




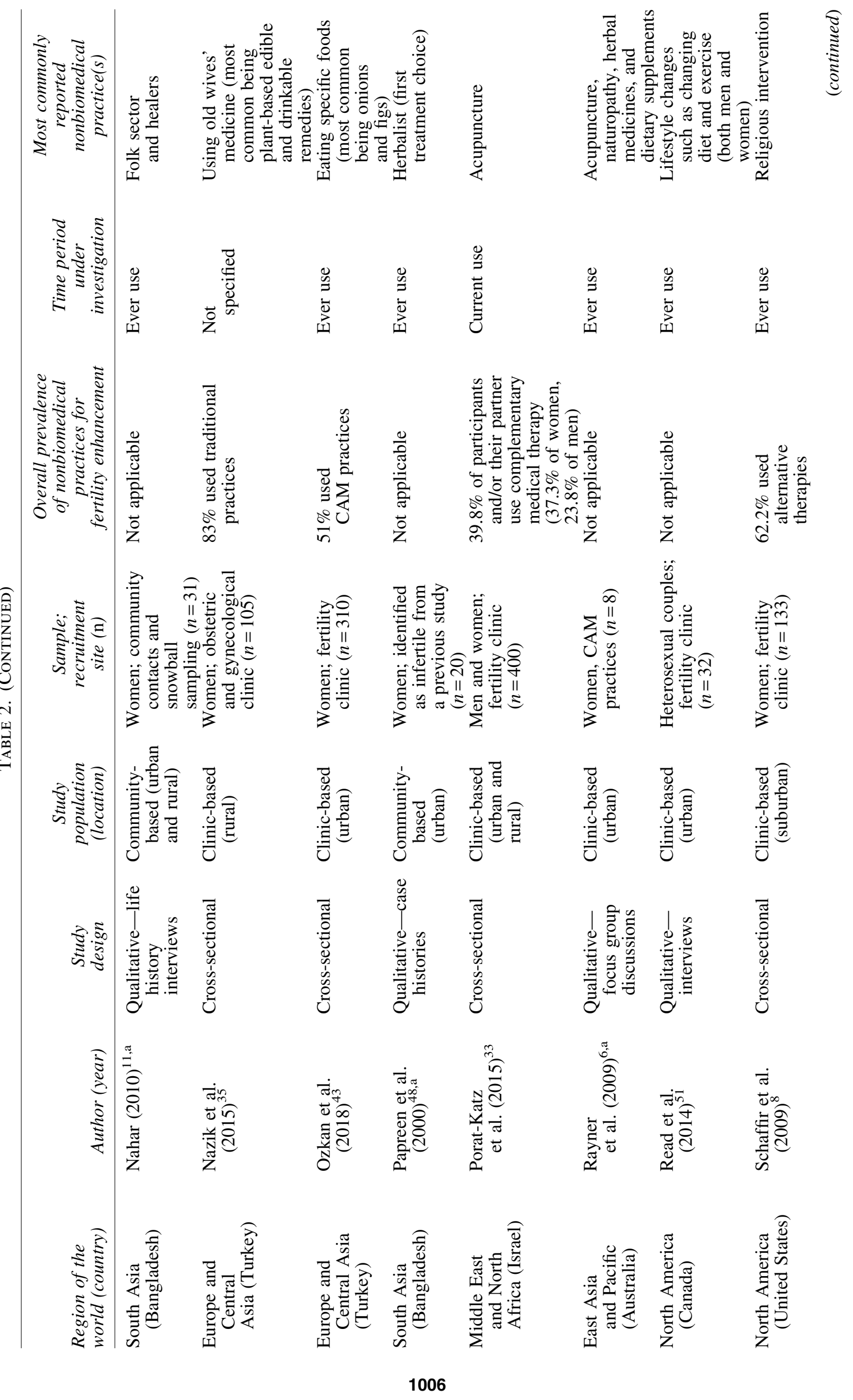




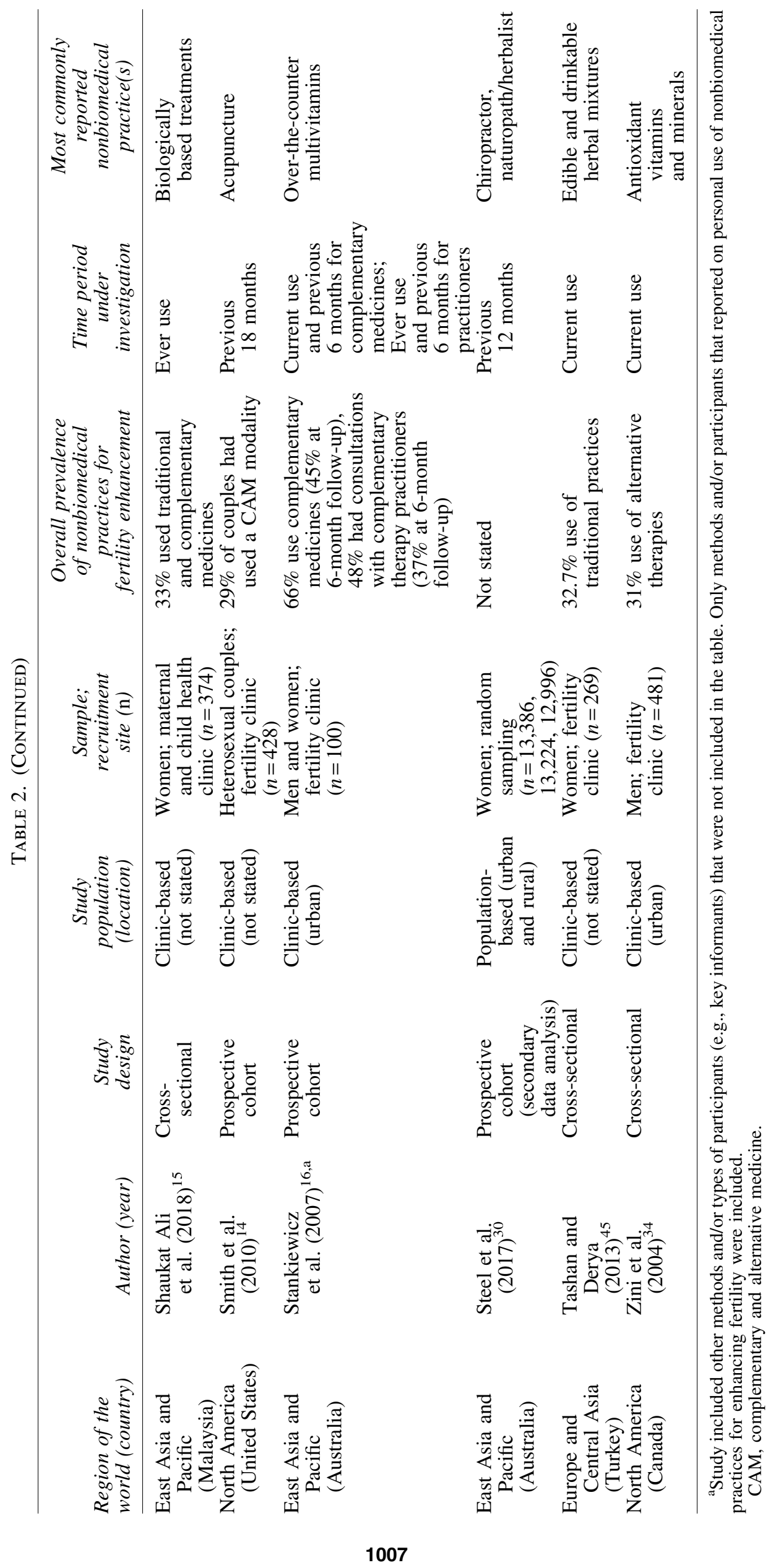




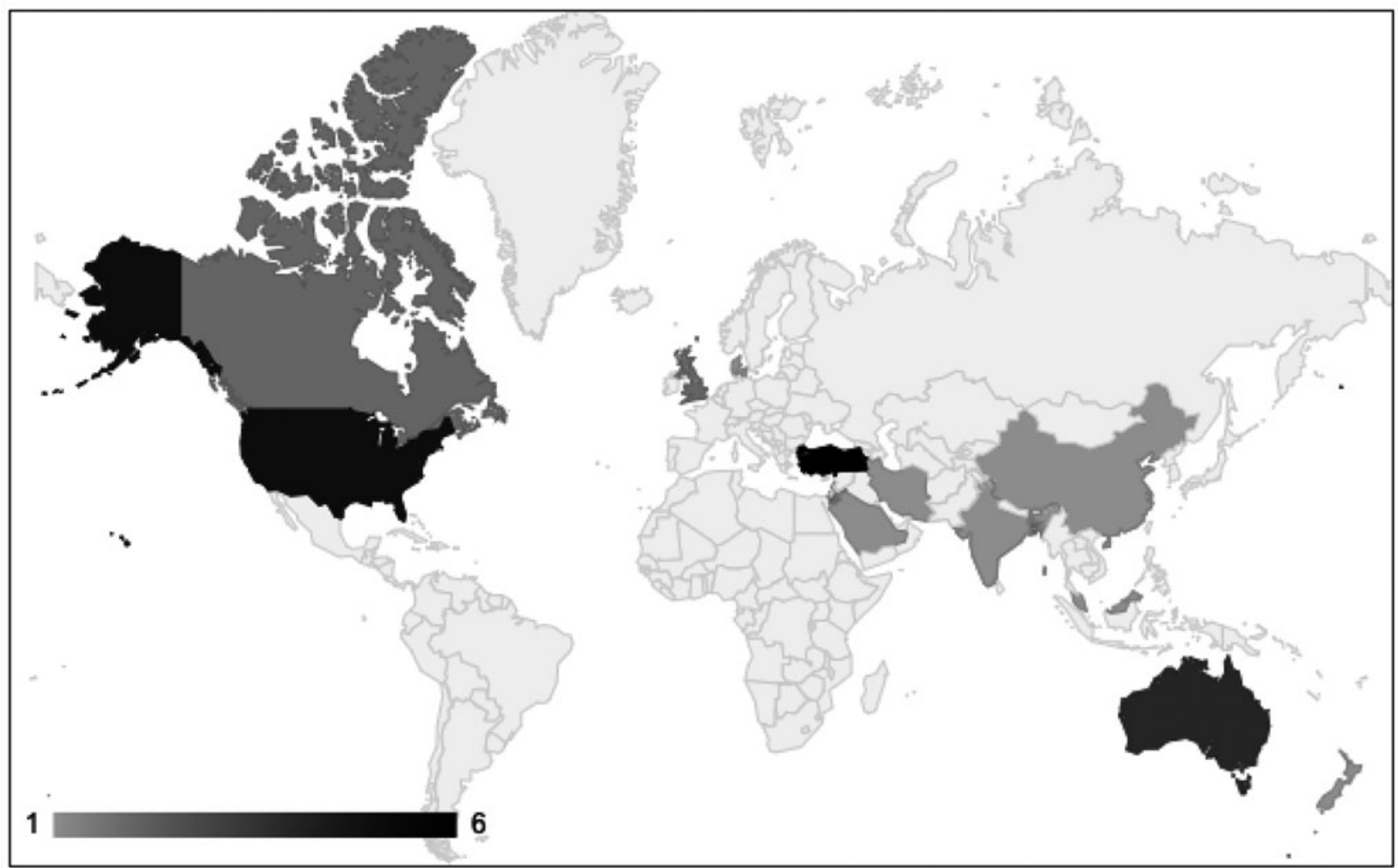

FIG. 2. Location of studies included in scoping review with number of studies indicated by intensity of shading.

comprehensive list of specific practices or groups of practices reported, the number of studies in which any use of a given practice was reported, and the regions in which the studies were conducted.

Biologically based treatments. Thirty-one studies reported some use of biologically based treatments, the most of any category $6,8,11,12,14-17,24-29,31-36,42-52$ with 18 studies reporting a biologically based treatment as a most commonly used practice among participants. ${ }^{6,15-17,24-27,29,34-36,43-48}$ More specifically, herbal medicine was the most common practice in the greatest number of studies $(n=8) 6,24,26,35,36,45,47,48$ and any use of herbal medicine was reported in nearly all studies. More than half of the studies that reported herbal medicine use did not provide details on types of herbs used, whereas nine studies reported names of individual herbs used, ${ }^{16,24,26,27,35,36,43,46,49}$ five studies reported use of Chinese herbs, ${ }^{12,24,26,46,51}$ and one study reported use of Brazilian herbs. ${ }^{6}$ Administration of herbal medicine varied and included oral consumption, ${ }^{24,29,45,47,49,52}$ external applications, ${ }^{43,49}$ intravaginal, ${ }^{36,43,49}$ and sitting over steam/vapor. ${ }^{36,43,45}$ All studies reporting nonoral applications of herbal or nonherbal medicines were conducted in Turkey. Nonherbal dietary supplements, such as vitamins, minerals, and antioxidants were also widely used and the most common practice in six studies. $6,16,29,34,44,46$ Some studies reported on specific supplements of which the following were commonly assessed and reported: vitamins B12, C, D, and E, folic acid, selenium, zinc, and fish oils. ${ }^{16,24,27,29,34}$ Hanna et al. analyzed posts in a men's online infertility discussion forum board and found that vitamins were among the most frequently discussed topic with many contributors describing their own, sometimes extensive, vitamin regime used to enhance their fertility. ${ }^{44}$

Religious and spiritual interventions. Over half of the studies reported some and often multiple use of religious and spiritual interventions explicitly with the belief that such practices would enhance fertility $(n=17)^{8,11,12,17,25,26,32,35,36,42,43,46-51}$ Seven studies reported these types of practices as a most commonly used practice. $8,17,26,32,42,49,50$ Several studies described these practices generally as faith/spiritual/religious healing or therapy, ${ }^{11,17,25,26,42,46,48,49}$ while prayer was the most common specific practice reported, which was described in studies as prayer, supplication, intercession, and citing religious text. $8,12,17,32,46,50,51$ For example, authors of a qualitative study conducted with men with infertility in China reported that "participants went to the temples and prayed to the gods of fertility on special occasions, such as the first and the fifteenth of the lunar month to bring about a favor from these gods." 12 Other common religious and spiritual practices mentioned in studies include using amulets ${ }^{11,35,36,42,43,49}$ and visiting holy or spiritual sites. ${ }^{35,42,43,49}$

Manipulative and body-based methods. Twenty studies reported some use of manipulative and body-based methods. ${ }^{6,8,12,14-16,25,26,30-33,35,36,42,43,46,49,51,52}$ Four studies reported a manipulative and body-based method as a most commonly used practice including reflexology, ${ }^{25,31}$ 


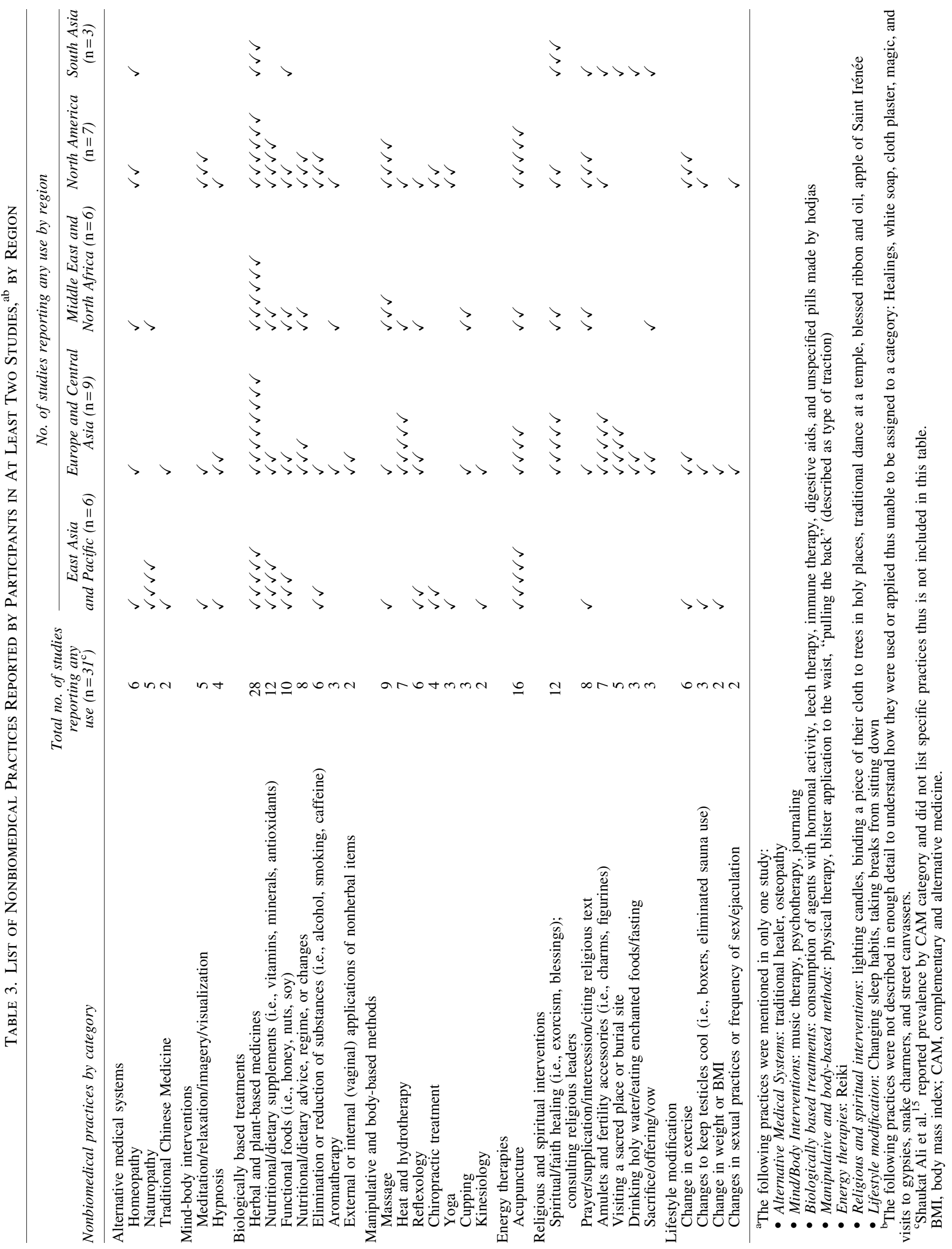


chiropractic treatment, ${ }^{30}$ and massage. ${ }^{52}$ Among all manipulative and body-based methods reported, massage was noted in the greatest number of studies. $8,12,14,26,32,33,43,46,52$ Use of heat and/or hydrotherapy, such as visiting thermal spas/baths and applying hot vacuum glasses to the groin area, was reported in multiple studies, primarily from Turkey. ${ }^{35,36,42,43,49}$ Furthermore, a qualitative study by Becker et al., conducted with participants from the Latino community in San Francisco, explained that many participants received an infertility diagnosis of a "cold womb" from practitioners of humoral medicine and subsequently used massage, hot tea, and staying warm at home to treat this condition.", 52

Energy therapies. Just over half of the studies $(n=17)$ reported some use of energy therapies $6,8,12,14-16,25,26,28-$ $31,33,43,44,46,51$ with four studies reporting acupuncture as a most commonly reported practice. ${ }^{6,14,33,46}$ Only one study reported use of another energy therapy-Reiki. ${ }^{43}$

Alternative medical systems. Use of alternative medical systems was reported in 13 studies. ${ }^{6,11,15,16,24,25,29-31,33,42,46,51}$ Three studies reported an alternative medical system as a most commonly used practice (naturopath ${ }^{6,30}$ and traditional heal$\mathrm{er}^{42}$ ). Homeopathy was the alternative medical system reported in the greatest number of studies. ${ }^{11,24,31,33,46,51}$

Lifestyle modification. Lifestyle modifications were reported in one quarter of the studies $(n=8) .8,12,28,29,43,44,46,51$ Only two studies, both conducted in North America, reported practices categorized as lifestyle modification as the most commonly reported, specifically noting changes in amount or intensity of exercise. ${ }^{28,51}$ Three studies reported lifestyle changes made by men to improve sperm quality, including wearing looser underwear ${ }^{8,44}$ and avoiding saunas. ${ }^{12}$

Mind/body interventions. Mind/body interventions were among the least reported practices with fewer than $25 \%(n=7)$ of the studies reporting any use of these methods $6,14,15,25,43,46,51$ and zero studies reporting these practices as the most commonly used. Meditation along with relaxation, imagery, and visualization were the most commonly reported practices in this category. $6,14,43,46,51$
Regional differences. Homeopathy, herbal and plantbased medicines, functional foods, and prayer were the only four practices reported in at least one study across all five regions while nutritional/dietary supplements, massage, reflexology, acupuncture, and spiritual/faith healing were reported in studies from four regions. Use of alternative medical systems was heavily reported in studies in East Asia and Pacific. Religious and spiritual interventions were reported in the highest concentration of studies in Europe and Central Asia, Middle East and North Africa, and South Asia. No studies conducted in the Middle East and North Africa and South Asia reported use of mind/body interventions or lifestyle modifications. Furthermore, no studies in South Asia reported use of manipulative and body-based methods or energy therapies.

\section{Terminology and format of survey question(s)}

We examined the terminology researchers used when referring to nonbiomedical practices in their survey instrument question(s). For this particular aspect, we only examined the eight studies for which we had exact wording of their question(s). Variation in terminology was found; however, four studies used similar terminology by referring to the practices as complementary therapies, ${ }^{25,26} \mathrm{CAM}$ therapies, ${ }^{27}$ and CAM. ${ }^{17}$ Notably, one study did not use a specific term when asking about nonbiomedical practices but rather asked participants to "please answer what you have done specifically to get pregnant." 8

We further explored how researchers asked participants about types of nonbiomedical practices used. Most studies used a close-ended question(s), often with an "other" option for participants to list practices not provided in predetermined response options. Twelve studies provided a list of the specific nonbiomedical practices examined in their survey instrument either as response options to a single question or as multiple individual questions. $8,16,17,25-28,30-34$ The number of specific practices examined in a given study ranged from 5 to 13 , which we then categorized using the same classification structure that we used earlier. Biologically based treatment practices were examined in the greatest number of studies $(n=11)$ (Fig. 3), with herbal medicine being the most common practice asked about. ${ }^{8,16,17,25-28,31-34}$
FIG. 3. Number of studies that asked participants about use of at least one practice within a given category $(n=12)$.

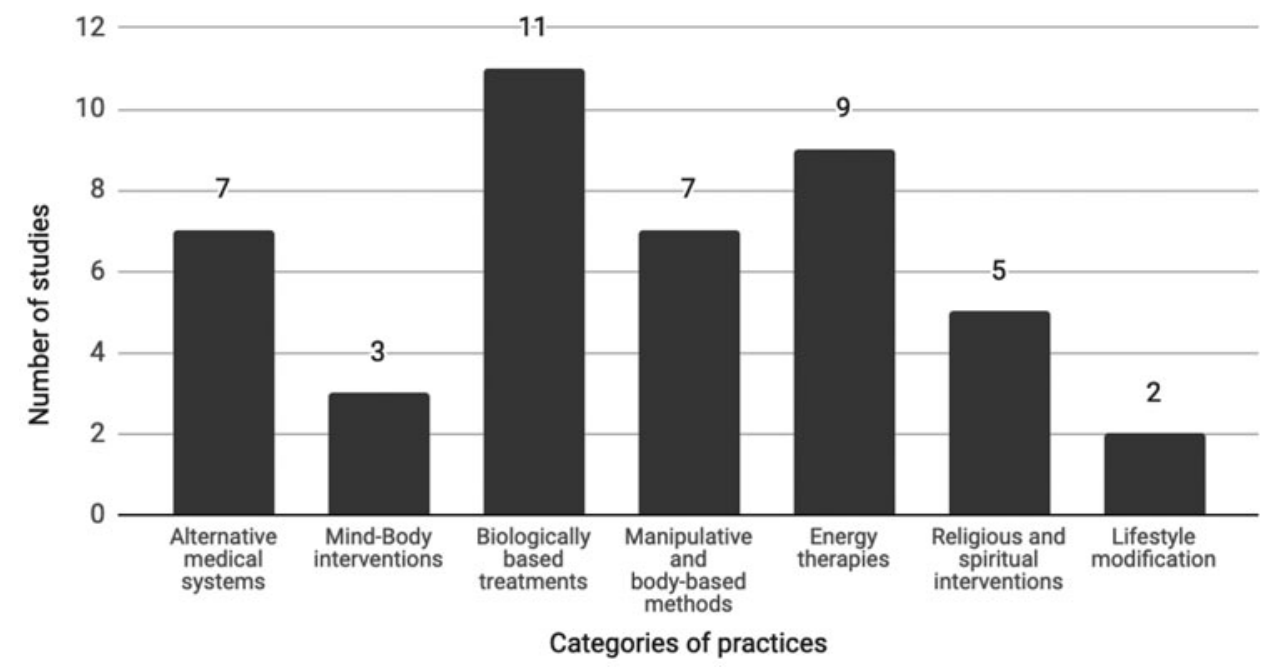


Very few studies asked about mind/body intervention or lifestyle modification practices. Two studies asked only about practices from a single category, of which both were conducted with men and focused exclusively on biologically based treatments. ${ }^{27,34}$ Only one study asked about practices from six categories ${ }^{25}$ and no studies asked about practices from all seven categories.

\section{Discussion}

Our scoping review had three primary aims: (1) to describe the scope and coverage of the body of literature on nonbiomedical practices used for enhancing fertility, (2) to summarize and map the existing evidence on the extent and types of nonbiomedical practices used, and (3) to examine how the research on this topic has been conducted. We start our discussion with a brief summary of the existing evidence on the extent and types of nonbiomedical practices used to enhance fertility but then allocate the remainder of our discussion to considering gaps and limitations in the research and putting forth recommendations for improving future research.

A total of 32 articles published between January 2000 and November 2018 were included in this scoping review representing research from 16 unique countries in five world regions. Most studies recruited participants from urban and suburban fertility clinics and found prevalence rates of nonbiomedical practice use for fertility enhancement ranging from $8 \%$ to $83 \%$, which is similar to findings from the 2011 review by Rayner et al. ${ }^{9}$ A wide variety of practices were reported across studies with multiple practices reported in each of the seven categories that we used to map our results. Biologically based treatments, particularly herbal medicines and nutritional/dietary supplements, was the most commonly reported category, reinforcing the urgent need for evidence-based information on the effectiveness and safety of these substances and the possibility of contraindication with ART, which, to date, is very limited. ${ }^{18,19} \mathrm{We}$ also found the use of spiritual and religious interventions and energy therapies, specifically acupuncture, reported in multiple studies. When comparing results by region, we found that some practices cut across all regions whereas other practices are only reported in certain regions. Understanding cultural and regional differences related to fertility enhancement is particularly important for practitioners working in certain settings and/or with immigrant or refugee communities whose beliefs and practices might differ from those of the practitioners.

In thinking about gaps and limitations in the research, we started by considering who is represented in the data and, more importantly, who is missing. First, two regions of the world were not represented in our scoping review-Latin America and Caribbean and sub-Saharan Africa. Furthermore, only 16 unique countries were represented and 15 of the 32 studies came from just three countries. This could be, in part, due to only including articles published in English. It could also highlight disparities in the prioritization of infertility research across regions and countries. With the lack of representation of certain regions and countries there are likely practices that were not captured in our review.

The vast majority of studies in our review were conducted in cities resulting in an under-representation of rural resi- dents, which could impact prevalence rates given that $\mathrm{Gu}-$ nay et al. reported use of traditional practices among rural residents in Turkey as twice as a high as urban residents. ${ }^{42}$ Despite most studies being conducted in urban areas, recruitment was largely through fertility clinics, which often serve both urban and rural residents due to a lack of such clinics in rural areas. Thus, it is possible that rural residents were represented in many of the studies but this information was not reported and/or results were not assessed by urban/rural status. We recommend that, at a minimum, the urban/rural status of participants be reported even when the study itself is conducted in an urban area and that results be stratified by this variable. The recruitment of participants from fertility clinics has other implications, including limiting the generalizability of findings on the use of nonbiomedical practices to only those that are subfertile and willing to access biomedical treatment for infertility. There is a clear need for more community and population-based studies that include a less homogenous sample.

Less than half of the studies in our review included men with only a few studies focusing exclusively on male experiences. This finding might be due, in part, to sociocultural norms in some settings that assign responsibility for infertility to women or to the fact that these norms often lead to women being subjected to more severe consequences than men, including neglect, humiliation, domestic violence, and divorce $;, 53$ both of these factors could result in prioritizing research on women's experiences with infertility. Despite social misconceptions, male factor infertility is the sole or combined factor in nearly $50 \%$ of infertility cases, ${ }^{54}$ highlighting the importance in capturing men's unique experiences with infertility. For example, the study by Hanna et al., which analyzed posts from an online forum for male infertility, identified a range of lifestyle modifications men were making as a means to enhancing sperm quality ${ }^{44}$; however, we found that these practices were not included as response options in the studies in our review even when men were included in the sample.

Lesbian, gay, bisexual, transgender, and queer (LGBTQ) individuals and couples face unique fertility challenges; despite this, an article by Fledderjohann and Barnes exposes the failure of infertility data to include all social groups, including LGBTQ, affected by infertility through two processes-sampling and survey instrument design. ${ }^{55}$ We found support for this argument with an overwhelming lack of inclusion and/or analysis of nonbiomedical practice use by LGBTQ individuals and couples. According to one study, one in six American LGBTQ adults avoid health care due to anticipated discrimination, ${ }^{56}$ further stressing the unique challenges that this subgroup faces, which could influence their engagement in nonbiomedical practices in ways that are distinct from heterosexual individuals and couples.

When considering prevalence and comparability across studies, the selection of the time period for which participants are asked about their use of nonbiomedical practices is important, especially considering nonbiomedical practices are used in different ways-concurrently, consecutively, or alternatively to conventional biomedicine. ${ }^{13}$ There is value in understanding current and ever use of nonbiomedical practices and, when feasible, collecting a detailed history of use, which could be done using a modified life history 
calendar that collects a complete history of an individual or couple's use of nonbiomedical practices as well as biomedical treatment throughout the duration of trying to conceive. This strategy would also allow researchers to measure duration and frequency of use of specific practices, something that was lacking in most of the studies that we reviewed, which was surprising given that many studies analyzed associations between "users" of nonbiomedical practices and predictor and outcome variables without accounting for duration or frequency of use. Furthermore, very few studies reported on the number of nonbiomedical practices in which participants were engaging, another unfortunate omission, and an important area to consider for future research particularly regarding its impact on quality of life and well-being.

We also examined survey instrument question(s) used by the studies in our review to inquire about participants' use of nonbiomedical practices to consider if and how the wording and format could impact comparability across studies as well as participant responses. We found that researchers often used terms similar to "complementary and alternative medicine" when asking about the use of nonbiomedical practices, despite studies reporting that individuals conceptualize this term differently. ${ }^{15,57,58}$ Schaffir et al. ${ }^{8}$ avoided labeling the practices by simply asking participants to specify what they have done specifically to get pregnant. This phrasing helps avoid potential differences in how participants interpret and respond to a question about nonbiomedical practices and allows for a broader understanding of the practices that participants are engaging in beyond the boundaries of CAM.

Given the extensive number of nonbiomedical practices, it is not feasible to ask participants about their use of each individual practice thus, unless using open-ended questions, researchers must decide which practices to ask about in their survey instruments, which could bias results even when an "other" response option is provided. Among the 12 studies in our review that provided the list of practices examined in their survey instrument, most examined practices from four or fewer of the seven categories that we used in mapping our results. This highlights a challenge and limitation of our scoping review in that we excluded studies focusing on a single practice such as herbal medicine but did not exclude studies focusing on a limited number of categories of practices such as biologically based treatments or lifestyle modifications. Clearly, if the objective of a study is to examine a certain practice or collection of practices, more targeted questioning is warranted. However, when the aim of the study is to identify the use of all nonbiomedical practices used to enhance fertility, then the survey question(s) and response options need to be carefully considered to allow for this information to be accurately and comprehensively collected. A rigorous, largely qualitative process was used to develop the CAM questionnaires used in the National Health Interview Survey ${ }^{59}$ and could potentially serve as a model. Furthermore, we must find ways to capture not only well-studied and/or well-documented practices but also current trends and fads such that researchers and practitioners can best support individuals with accurate and relevant information and education. Online forum analyses, such as the study by Hanna et al. on male infertility, ${ }^{44}$ might be particularly useful in identifying trends and fads.

\section{Conclusions}

This scoping review identified a wide variety of nonbiomedical practices that many individuals around the world use to enhance their fertility. However, it also uncovered limitations and variation in what, how, and from whom information is being collected and reported, making comparisons across studies and regions challenging, which, in turn, limits our ability to draw meaningful conclusions about the use of nonbiomedical practices that could inform subsequent research and practice. Based on our findings, we provide recommendations for strengthening research on the use of nonbiomedical practices for fertility enhancement such that it is more comprehensive, inclusive, and informative, which, we believe, can ultimately lead to equipping individuals and couples with information they need to make informed decisions about their use of nonbiomedical practices to enhance their fertility.

\section{Acknowledgments}

The authors thank Anne Beschnett, Research and Instruction Librarian at St. Catherine University, for her technical guidance in conducting a scoping review.

\section{Author Disclosure Statement}

No competing financial interests exist.

\section{Funding Information}

This work was supported by the GHR Foundation through the Academic Excellence Grant awarded to St. Catherine University (grant \#9512).

\section{References}

1. Gerrits T, Van Rooij F, Esho T, et al. Infertility in the Global South: Raising awareness and generating insights for policy and practice. Facts Views Vis Obgyn 2017;9: 39-44.

2. Boivin J, Bunting L, Collins JA, Nygren KG. International estimates of infertility prevalence and treatment-seeking: Potential need and demand for infertility medical care. Hum Reprod 2007;22:1506-1512.

3. Gurunath S, Pandian Z, Anderson R, Bhattacharya S. Defining infertility-A systematic review of prevalence studies. Hum Reprod Update 2011;17:575-588.

4. Rouchou B. Consequences of infertility in developing countries. Perspect Public Health 2013;133:174-179.

5. Hubens K, Arons AMM, Krol M. Measurement and evaluation of quality of life and well-being in individuals having or having had fertility problems: A systematic review. Eur J Contracept Reprod Health Care 2018;23:441450.

6. Rayner JA, McLachlan HL, Forster DA, Cramer R. Australian women's use of complementary and alternative medicines to enhance fertility: Exploring the experiences of women and practitioners. BMC Complement Altern Med 2009;9:52.

7. Dierickx S, Balen J, Longman C, et al. 'We are always desperate and will try anything to conceive': The convoluted and dynamic process of health seeking among women with infertility in the West Coast Region of The Gambia. PLoS One 2019;14:e0211634. 
8. Schaffir J, McGee A, Kennard E. Use of nonmedical treatments by infertility patients. J Reprod Med 2009;54: 415-420.

9. Rayner J-A, Willis K, Burgess R. Women's use of complementary and alternative medicine for fertility enhancement: A review of the literature. J Altern Complem Med 2011;17:685-690.

10. Sarkar S, Gupta P. Socio-demographic correlates of women's infertility and treatment seeking behavior in India. J Reprod Infertil 2016;17:123-132.

11. Nahar P. Health seeking behaviour of childless women in Bangladesh: An ethnographic exploration for the special issue on: Loss in child bearing. Soc Sci Med 2010;71: 1780-1787.

12. Lee TY, Chu TY. The Chinese experience of male infertility. West J Nurs Res 2001;23:714-725.

13. van Balen F, Gerrits T. Quality of infertility care in poorresource areas and the introduction of new reproductive technologies. Hum Reprod 2001;16:215-219.

14. Smith JF, Eisenberg ML, Millstein SG, et al. The use of complementary and alternative fertility treatment in couples seeking fertility care: Data from a prospective cohort in the United States. Fertil Steril 2010;93:2169-2174.

15. Shaukat Ali R, Gnanasan S, Farooqui M. Women's perspectives towards traditional and complementary medicine used to conceive, during pregnancy and the postpartum period. Complement Ther Clin Pract 2018;30:109-115.

16. Stankiewicz M, Smith C, Alvino H, Norman R. The use of complementary medicine and therapies by patients attending a reproductive medicine unit in South Australia: A prospective survey. Aust N Z J Obstet Gynaecol 2007;47: 145-149.

17. Ghazeeri GS, Awwad JT, Alameddine M, et al. Prevalence and determinants of complementary and alternative medicine use among infertile patients in Lebanon: A cross sectional study. BMC Complement Altern Med 2012;12:129.

18. Weiss DA, Harris CR, Smith JF. The use of complementary and alternative fertility treatments. Curr Opin Obstet Gynecol 2011;23:195-199.

19. Miner SA, Robins S, Zhu YJ, et al. Evidence for the use of complementary and alternative medicines during fertility treatment: A scoping review. BMC Complement Altern Med 2018;18:158.

20. Arksey H, O’Malley L. Scoping studies: Towards a methodological framework. Int J Soc Res Methodol 2005;8: 19-32.

21. Levac D, Colquhoun H, O'Brien KK. Scoping studies: Advancing the methodology. Implement Sci 2010;5:69.

22. Peters MD, Godfrey CM, Khalil H, et al. Guidance for conducting systematic scoping reviews. Int J Evid Based Healthc 2015;13:141-146.

23. Munn Z, Peters MDJ, Stern C, et al. Systematic review or scoping review? Guidance for authors when choosing between a systematic or scoping review approach. BMC Med Res Methodol 2018;18:143.

24. Charaf S, Wardle JL, Sibbritt DW, et al. Women's use of herbal and alternative medicines for preconception care. Aust N Z J Obstet Gynaecol 2015;55:222-226.

25. Coulson C, Jenkins J. Complementary and alternative medicine utilisation in NHS and private clinic settings: A United Kingdom survey of 400 infertility patients. J Exp Clin Assist Reprod 2005;2:5.

26. Bardaweel SK, Shehadeh M, Suaifan GA, Kilani MV. Complementary and alternative medicine utilization by a sample of infertile couples in Jordan for infertility treatment: Clinics-based survey. BMC Complement Altern Med 2013;13:35.

27. Bardaweel SK. Alternative and antioxidant therapies used by a sample of infertile males in Jordan: A cross-sectional survey. BMC Complement Altern Med 2014;14:244.

28. Domar AD, Conboy L, Denardo-Roney J, Rooney KL. Lifestyle behaviors in women undergoing in vitro fertilization: A prospective study. Fertil Steril 2012;97:697-701.e1.

29. Gormack AA, Peek JC, Derraik JG, et al. Many women undergoing fertility treatment make poor lifestyle choices that may affect treatment outcome. Hum Reprod 2015;30: 1617-1624.

30. Steel A, Adams J, Sibbritt D. The characteristics of women who use complementary medicine while attempting to conceive: Results from a nationally representative sample of 13,224 Australian women. Womens Health Issues 2017; 27:67-74

31. Boivin J, Schmidt L. Use of complementary and alternative medicines associated with a $30 \%$ lower ongoing pregnancy/live birth rate during 12 months of fertility treatment. Hum Reprod 2009;24:1626-1631.

32. Dehghan M, Mokhtarabadi S, Heidari FG. Complementary and alternative medicine usage and its determinant factors among Iranian infertile couples. J Complement Integr Med 2018;15. DOI: $10.1515 /$ jcim-2017-0138.

33. Porat-Katz A, Eldar-Geva T, Kahane A, et al. Use of complementary medical therapies by Israeli patients undergoing in vitro fertilization. Int J Gynaecol Obstet 2015; 129:133-137.

34. Zini A, Fischer MA, Nam RK, Jarvi K. Use of alternative and hormonal therapies in male infertility. Urology 2004; 63:141-143.

35. Nazik E, Apay S, Ozdemir F, Nazik H. Traditional Practices of Turkish infertile women: An example from a rural county. Coll Antropol 2015;39:21-25.

36. Ayaz S, Efe SY. Traditional practices used by infertile women in Turkey. Int Nurs Rev 2010;57:383-387.

37. Institute of Medicine (U.S.). Committee on the Use of Complementary and Alternative Medicine by the American Public. Complementary and Alternative Medicine in the United States. Washington, DC: The National Academies Press, 2005.

38. Wieland LS, Manheimer E, Berman BM. Development and classification of an operational definition of complementary and alternative medicine for the Cochrane collaboration. Altern Ther Health Med 2011;17:50-59.

39. Millstine D. Types of complementary and alternative medicine. MSD Manual Professional Version. Online document at: www.msdmanuals.com/professional/special-subjects/ integrative,-complementary,-and-alternative-medicine/typesof-complementary-and-alternative-medicine, accessed May $5,2020$.

40. Tricco AC, Lillie E, Zarin W, et al. PRISMA extension for scoping reviews (PRISMA-ScR): Checklist and explanation. Ann Intern Med 2018;169:467-473.

41. The World Bank Group. World Bank Country and Lending Groups. The World Bank Group. Online document at: https://datahelpdesk.worldbank.org/knowledgebase/articles/ 906519-world-bank-country-and-lending-groups, accessed February 2, 2020.

42. Gunay O, Cetinkaya F, Nacar M, Aydin T. Modern and traditional practices of Turkish infertile couples. Eur J Contracept Reprod Health Care 2005;10:105-110. 
43. Ozkan FS, Karaca A, Sarak K. Complementary and alternative medicine used by infertile women in Turkey. Afr J Reprod Health 2018;22:40-48.

44. Hanna E, Gough B, Hudson N. Fit to father? Online accounts of lifestyle changes and help-seeking on a male infertility board. Sociol Health Illn 2018;40:937-953.

45. Timur Tashan S, Aksoy Derya Y. Traditional practices used by the infertile women to become pregnant and their effects on the quality of life. Int J Nurs Pract 2013;19:516-522.

46. Clark NA, Will MA, Moravek MB, et al. Physician and patient use of and attitudes toward complementary and alternative medicine in the treatment of infertility. Int J Gynaecol Obstet 2013;122:253-257.

47. Guntupalli AM, Chenchelgudem P. Perceptions, causes and consequences of infertility among the Chenchu tribe of India. J Reprod Infant Psychol 2004;22:249-259.

48. Papreen N, Sharma A, Sabin K, et al. Living with infertility: Experiences among Urban slum populations in Bangladesh. Reprod Health Matters 2000;8:33-44.

49. Edirne T, Arica SG, Gucuk S, et al. Use of complementary and alternative medicines by a sample of Turkish women for infertility enhancement: A descriptive study. BMC Complement Altern Med 2010;10:7.

50. Al-Jaroudi DH. Beliefs of subfertile Saudi women. Saudi Med J 2010;31:425-427.

51. Read SC, Carrier ME, Whitley R, et al. Complementary and alternative medicine use in infertility: Cultural and religious influences in a multicultural Canadian setting. J Altern Complement Med 2014;20:686-692.

52. Becker G, Castrillo M, Jackson R, Nachtigall RD. Infertility among low-income Latinos. Fertil Steril 2006;85: 882-887.
53. Stellar C, Garcia-Moreno C, Temmerman M, van der Poel S. A systematic review and narrative report of the relationship between infertility, subfertility, and intimate partner violence. Int J Gynaecol Obstet 2016;133:3-8.

54. de Kretser DM. Male infertility. Lancet 1997;349:787-790.

55. Fledderjohann J, Barnes LW. Reimagining infertility: A critical examination of fertility norms, geopolitics and survey bias. Health Policy Plan 2018;33:34-40.

56. Casey LS, Reisner SL, Findling MG, et al. Discrimination in the United States: Experiences of lesbian, gay, bisexual, transgender, and queer Americans. Health Serv Res 2019; 54 Suppl 2:1454-1466.

57. Chatterjee A. A qualitative analysis of the naming process of complementary and alternative medicine by chronically ill patients. Complement Ther Med 2018;41:306-310.

58. Bishop FL, Yardley L, Lewith GT. Treat or treatment: A qualitative study analyzing patients' use of complementary and alternative medicine. Am J Public Health 2008;98: 1700-1705.

59. Stussman BJ, Bethell CD, Gray C, Nahin RL. Development of the adult and child complementary medicine questionnaires fielded on the National Health Interview Survey. BMC Complement Altern Med 2013;13:328.

Address correspondence to: Carie Muntifering Cox, PhD, MPH Consultant 33 Rue Albert 1er Luxembourg L-1117 Luxembourg

E-mail: cjmuntifering@gmail.com 